\title{
Experimental Measurement of Thermal Conductivities in a Thin Heterogeneous Structure of Thermal Diodes
}

\author{
Jieyang Zhou ${ }^{1,2}$, Zhe Wang ${ }^{1, *}$, and Yun Wang ${ }^{2}$ \\ ${ }^{1}$ Department of Energy and Power Engineering, Tsinghua University, 100084, Beijing, China \\ ${ }^{2}$ Renewable Energy Resources Lab, Department of Mechanical and Aerospace Engineering, University of California, Irvine, 92697-3975, \\ CA, United States
}

\begin{abstract}
Thermal diode has a wide application in the field of thermal management and thermal control. This article reports experimental results about measurement of the thermal conductivities of a novel thin layer (the thickness is about $0.3 \mathrm{~mm}$ ) for thermal diode applications. The layer consists printing paper, nylon mesh and liquid water, which are sealed between two pieces of aluminum, thus has a heterogeneity sublayers structure. It is shown that the thermal conductances are different in the two opposite throughplane directions. At $75{ }^{\circ} \mathrm{C}$, the thermal conductivity is $0.457 \mathrm{~W} / \mathrm{mK}$ in the conductive direction, more than 3 times larger than that in the opposite direction $(0.133 \mathrm{~W} / \mathrm{mK})$. This phenomenon is due to the one-direction flow of working fluid. The thermal performance is dependent on the operating temperature and liquid water content in the structure.
\end{abstract}

\section{Introduction}

Thermal diode devices and thermal rectification devices have broad applications in the field of thermal control and management. However, materials in nature usually share the same thermal conductivity in any opposite directions. This limits multi-function thermal design which enables control of heat transfer directions. For example, in some cases, thermal components are required to direct heat from the surroundings to the inside, with preventing heat escape to the outside. And in other cases, materials that can allow heat flow from within equipment to the surroundings and avoid the heat from environment to enter are demanded. At this time, isotropic materials in thermal conductivity no longer meet the requirements. Recently, with the development of thermal control and management, some special thermal components are produced to control the direction of heat transfer. Wang introduced a novel thin layer, which is based on heat pipe effect, for thermal diode applications [1], which can implement the above functions. As for thin layer of microscale thickness, the apparent conductance promoted by heat pipe is considerable [2].

Moreover, thermal properties are key to many applications, especially for thermal and power machines, like gas turbine, coal-fired boiler, internal combustion engine, fuel cell and more. Some unique properties, such as insulation, large conductance, high thermal capacity, large latent heat and anisotropic thermal conductivity, offer superior capabilities in these applications. Among them, thermal conductivity attracts more attention from people, as it's a basic parameter for thermal materials, and of great importance in extensive research fields.

Accurate measurements of these thermodynamic properties, like thermal conductivity, have always been an important research direction, not only for pure theoretical research, but also for experimental science and engineering. There are many experimental methods existing for measuring thermal conductivities, including steady-state methods, transient plane/line source method, modified transient plane/line source method, laser flash method, time-domain thermoreflectance method and more. Yue et al. designed a steady-state electro-Ramenthermal technique for measuring the thermal contact of CNTs [3]. Wang and Gundevia introduced a novel method to test the thermal conductivity and heat pipe effect in carbon papers [4].

In this paper, we designed a layer consisting of a printing paper and nylon mesh, and tested the thermal conductivities of the thermal diodes by using a selfdesigned experimental instrument. Testing under various conditions including temperature and water content was conducted.

\section{Experimental}

\subsection{Structure of Heterogeneous Layer}

In order to achieve a thermal diode, a thin layer with heterogeneous structure was developed. As shown in the Figure 1, the thermal anisotropy thin layer contained two pieces of idiosyncratic wettability sublayers, one hydrophilic printing paper (Aspen ${ }^{\circledR} 30$, Boise ${ }^{\circledR}$, USA)

*Corresponding author: zhewang@tsinghua.edu.cn 
and one hydrophobic nylon mesh. Both of them were cut into a wafer with a diameter of $38 \mathrm{~mm}$. Also, deionized water (CRYSTAL GEYSER ${ }^{\circledR}$, USA) was added into the thin layer as working fluid, which would circulate between the sublayers under the drive of capillary force. According to the wettability of sublayers, the printing paper worked as a liquid attracting element, and the nylon cloth worked as a liquid repelling element in the layer. Besides, two pieces of aluminium foil (UltraClean $^{\mathrm{TM}}$, Control Company, USA), in the diameter of 42 $\mathrm{mm}$, were stuck with $100 \%$ silicone sealant (Momentive Performance Materials Inc., USA) as a container of working liquid and sublayers to avoid the evaporation of water.

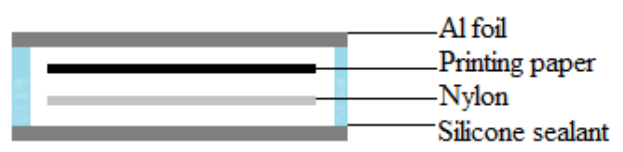

Fig. 1. The heterogeneous structure of the thermal diode

After the production, the wet-sealant samples were put into a vacuum chamber under vacuum for 24 hours to make sure that the silicone is dried enough and the samples do not contain any air in itself. The pieces of different materials were shown in the Figure $2 \mathrm{a}$, and the final sample was shown in the Figure $2 b$.

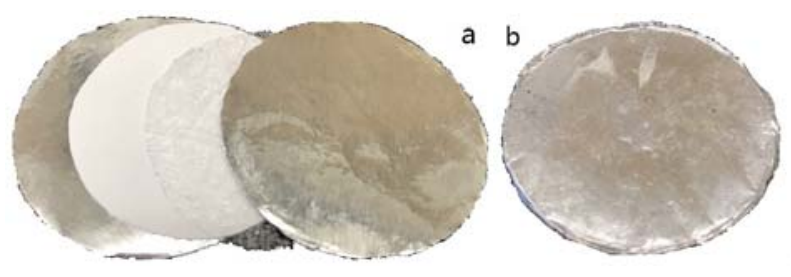

Fig. 2. a) Materials in the thermal diode (from left to right: Al foil, printing paper, nylon mesh, and $\mathrm{Al}$ foil); b) Final sample assembly.

And the water addition in the layer was weighed through an electronic balance (ACCULAB Sartorius Group, USA) during the preparation of the samples, and was reweighed again after testing. Based on the water content, samples were divided into three groups, I) 0 $\mathrm{wt} \%$ water, II) $15 \mathrm{wt} \%$ water (the mass fraction of the water is $15 \%$ ) and III) $40 \mathrm{wt} \%$ water.

\subsection{Experimental Methods}

The experimental apparatus was designed and improved based on the previous results [4], as shown in the Figure 3 , which could be divided into several parts, according to the different function during the experiment. The main part of the device contained an aluminium bar and a stainless steel bar, working as reference bars with three testing holes on each for measurement of temperature. By measuring the hole temperatures, and combining the thermal conductivities of reference materials, the heat flux $(q)$ through the layer could be determined.

At the top of the aluminium bar, four heating sources are embedded to control the working temperature of samples. By changing the heating power, the working temperatures of the samples were adjusted at three testing points with the same temperature difference: 45
${ }^{\circ} \mathrm{C}, 60{ }^{\circ} \mathrm{C}$ and $75{ }^{\circ} \mathrm{C}$. In experiment, it took one to four hours to reach a thermal steady state, when measurement was taken.

On the purpose of getting accurate experimental data, an insulation blanket was used to prevent heat loss during the testing. And all experiments were performed under the same external load to reduce the impact of contact thermal conductivities.

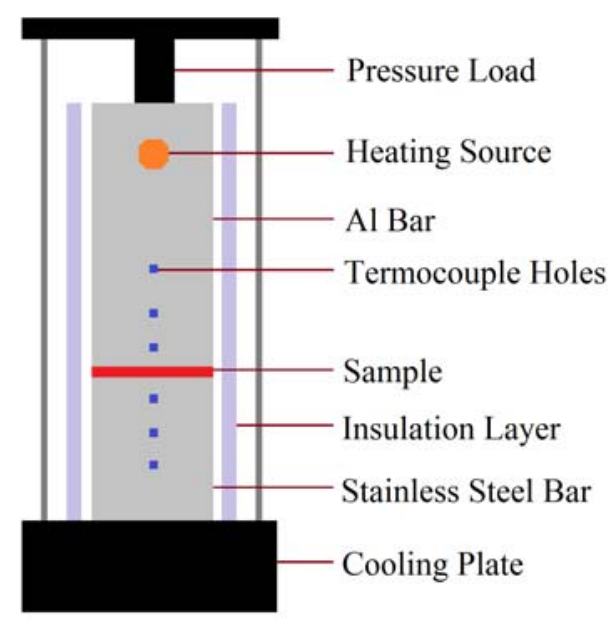

Fig. 3. Diagram of the experimental instrument

As explained above, measuring the temperatures of the testing holes could gave the heat flux of the layer. Simultaneously, the temperature difference $(\Delta T)$ between the two surfaces of the layer could also be calculated. According to the Fourier's law described below, the thermal resistance $(R)$ and the thermal conductivity $(k)$ of the sample would be determined by the heat flux, temperature difference and the thickness of the sample $(d)$ finally.

$$
k=d / R=d \cdot q / \Delta T
$$

This method was validated by testing the thermal conductivity of printing paper and compared with existing results. The testing result was $0.122 \mathrm{~W} / \mathrm{mK}$, which was close to others, like $0.125 \mathrm{~W} / \mathrm{mK}$ in Physical and Mathematical Tables (3rd edition) [5], and 0.105 $\mathrm{W} / \mathrm{mK}$ tested by Morikawa and Hashimoto [6], and more $[7,8]$. As the paper mentioned above were made of various pulp, the diameter of the fibre, the fibre type and the compactness of paper were totally different. Thus the conductivities were not exactly the same. All the data were listed in the Table 1 below.

Tab. 1. Thermal conductivities of paper in different references

\begin{tabular}{|l|c|c|c|}
\hline \multicolumn{1}{|c|}{ Type } & $\mathbf{k}(\mathbf{W} / \mathbf{m K})$ & Year & Ref. \\
\hline paper & 0.125 & 1979 & {$[5]$} \\
\hline $\begin{array}{l}\text { paper from Oji Paper } \\
\text { Co.Ltd. }\end{array}$ & 0.105 & 1998 & {$[6]$} \\
\hline cellulose paper & 0.152 & 2012 & {$[7]$} \\
\hline commercial copy paper & 0.0905 & 2012 & {$[8]$} \\
\hline
\end{tabular}

\section{Results and Discussion}


As a thermal diode, the performance of the heterogeneous layer changed a lot in the different heat transfer directions. Meanwhile, the water content and the working temperature also affected the heat conduction function of it.

\subsection{Thermal Conductivity under Temperature}

Figure 4 reflected the changing thermal conductivities of two different layers under varying temperature. The layer with high water content (40 wt $\%$ ) had an obviously increase of conductivity with the rise of temperature in the conductive direction, which meant the heat transfer was fast in this direction. Just as shown in the 'Conductive-40 wt\%' line, the thermal conductivity increased from $0.143 \mathrm{~W} / \mathrm{mK}$ at $45{ }^{\circ} \mathrm{C}$ to $0.457 \mathrm{~W} / \mathrm{mK}$ at $75{ }^{\circ} \mathrm{C}$, more than tripled in value. Meanwhile, it's equal to $0.336 \mathrm{~W} / \mathrm{mK}$ at $60{ }^{\circ} \mathrm{C}$, which demonstrated that the capacity of heat transmission was linearly growing in this range. These enhancements were mainly caused by the heat pipe effect between the sublayers, rather than the high thermal conductivity of the water itself.

However, in the opposite direction, this number only changed a little, from $0.113 \mathrm{~W} / \mathrm{mK}$ to $0.133 \mathrm{~W} / \mathrm{mK}$, only one third of the conductive one. But it's still larger than the no water group $(0 \mathrm{wt} \%, \sim 0.070 \mathrm{~W} / \mathrm{mK})$, about two times larger than that, and the positive one was more than 6 times larger than it. These phenomenon illustrated that the heat pipe effect was the main reason of the heightening in the conductive direction, instead of the adding water. What's more, a decrease was observed from $60{ }^{\circ} \mathrm{C}$ to $75{ }^{\circ} \mathrm{C}$ in the opposite direction, which was mainly caused by the measurement error.

As a control group, the thermal conductivity of the layer with $0 \mathrm{wt} \%$ working liquid only had small fluctuations under varying temperature, without significant tendency of variation.

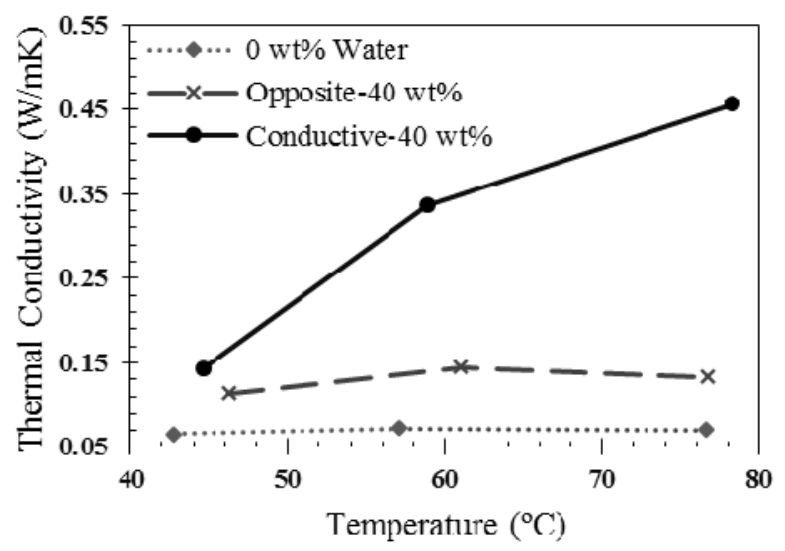

Fig. 4. Influence of temperature on the thermal conductivity with $40 \mathrm{wt} \%$ water and $0 \mathrm{wt} \%$ water

The phenomenon described above, was promoted by one-way heat pipe effect, and could be called as thermal diode. This effect was resulted from the transport and phase change of the working liquid. At the high temperature side, the liquid evaporated with absorbing heat, and turned into vapour. Then the resulting vapour transferred to the opposite side, condensed to liquid and released heat. Under capillary action and the effect of heterogeneity sublayer, the condensed work fluid flowed back to the evaporation side, restarting the cycle to transport the heat from the evaporation to the condensation sides constantly. Distinctly, the reflux of condensed liquid determined the strength of the heat pipe effect. Compared to nylon, paper was more hydrophilic, thus the performance of the paper near the heat source was much stronger than that of the nylon. According to these characteristics, the layers could act as thermal diodes, and control the direction of heat transfer.

\subsection{Impact of Water Content}

Water as a working liquid, the added dose would influence the strength of the heat pipe effect. Figure 5 included the curves of group ' $15 \mathrm{wt} \%$ water', which expressed completely different characteristics about temperature rising and reflected the importance of water content in the layers.

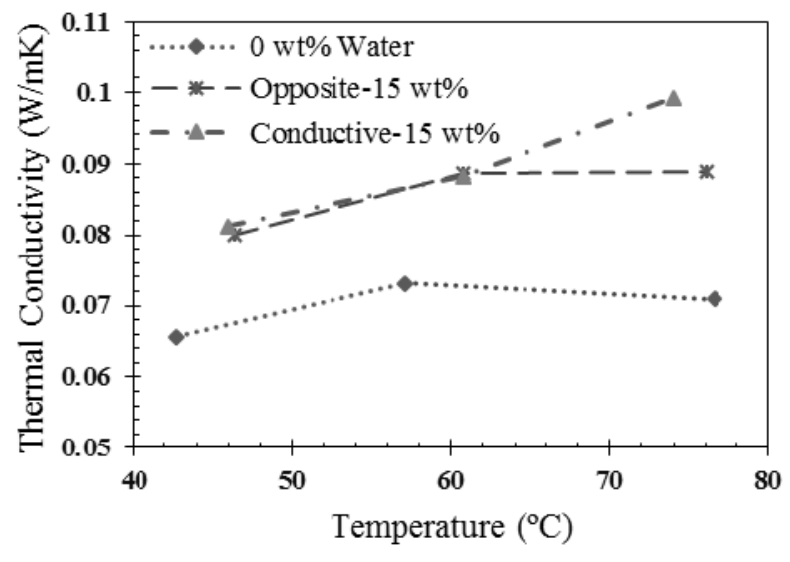

Fig. 5. Influence of temperature on the thermal conductivity with $15 \mathrm{wt} \%$ water and $0 \mathrm{wt} \%$ water

As discussed above, the thermal conduction properties fortified visibly, and the disparity between the different directions heightened as well, if the layer contained enough working liquid. Nevertheless, in these layers, although the thermal conductivity enhanced likewise, the difference between orientations was pretty small. Only at $75{ }^{\circ} \mathrm{C}$, the significant change was observed that it's $0.089 \mathrm{~W} / \mathrm{mK}$ in slow heat transfer direction, and increased to $0.099 \mathrm{~W} / \mathrm{mK}$ after reversed. As for $45^{\circ} \mathrm{C}$ and $60{ }^{\circ} \mathrm{C}$, the data almost didn't change at all, whether the layer was flipped or not. It's not noteworthy, but the growth of thermal conductivity still existed, from $0.080 \mathrm{~W} / \mathrm{mK}$ to $0.099 \mathrm{~W} / \mathrm{mK}$, almost had improved by $25 \%$. Additionally, the capacity of heat transmission of the $15 \mathrm{wt} \%$ water group was better than the $0 \mathrm{wt} \%$ group all the time under unequal temperatures.

Principally, these phenomenon was due to the water content in the layer. Without enough working fluid, the heat transfer cycle was hard to form, leading to a weak heat pipe effect. In spite of the noteless effect, the working liquid still enhanced the thermal conductivity of layers, on account of the higher conductivity of water than printing paper or nylon. Meanwhile, the heat pipe effect, forming between the micro structures in the 
sublayer, might make a contribution to growth. What's more, as the temperature rose, especially at high temperature, a weak thermal diode effect could be observed. High temperature improved the evaporation of the water, which played a key role in the formation of heat transfer cycle.

\section{Conclusion}

This paper presented an experimental study of the thermal conductivity of a novel thin layer combining heterogeneous structure. It is found that the thermal conductivity in one direction is more than 3 times larger than the opposite direction. The difference in the thermal conductance was caused by one-direction heat pipe effect, and was influenced by the working temperature. At $75{ }^{\circ} \mathrm{C}$, the thermal conductivities of conductive direction were more than 3 times than the opposite direction, with $40 \mathrm{wt} \%$ water. And the water content in the structure also influenced the ability as well. For 15 $\mathrm{wt} \%$ water content layers, the difference is only 1.25 times between two directions, much smaller than the 40 $\mathrm{wt} \%$ group. This type of thermal diodes is useful in various applications for thermal management and control.

\section{References}

1. Y. Wang, Thermal devices for controlling heat transfer, United States Patent No. US 2015/0226497 A1

2. Y. Wang, C. Y. Wang, J. Electrochem. Soc., 153, $1193(2006)$

3. Y. Yue, X. Huang, X. Wang, Phys. Lett. A, 40, 4144 (2010)

4. Y. Wang, M. Gundevia, Int. J. Heat Mass Transf., 60, 134 (2013)

5. T. M. Yarwood, F. Castle, G. R. Noakes, Physical and Mathematical Tables 3rd edition (Glasgow UK: The University Press, 1970)

6. J. Morikawa, T. Hashimoto, Polym. Int., 45, 207 (1998)

7. R. Lopatkiewicz, Z. Nadolny, P. Przybylek, IEEE $10^{\text {th }}$ International Conference on the Properties and Applications of Dielectric Materials, July 24-28, 2012

8. S. A. Lavrykov, B. V. Ramarao, Dry. Technol., 30, 297 (2012) 